\title{
Validación de Modismos de Visualización para la Socialización de Resultados de Evaluación en el Taller
}

\author{
Validation of Visual Idioms for Shared Assessment of Studio Course Results
}

- Bruno Perelli

Depto. Diseño, Universidad de Chile

bperelli@uchilefau.cl

Eduardo Hamuy

Depto. Diseño, Universidad de Chile

ehamuy@uchile.cl

\author{
Paola de la Sotta \\ Depto. Diseño, Universidad de Chile \\ psotta@uchile.cl
}

\begin{abstract}
A visualization artifact, aims at assisting a process of collective and individual analysis of assessment and learning outcomes from participants in a design studio course. A research by design approach supported by the Nested Model for Visualization Design and Validation framework was used. Display modes in Cartesian structure diagrams and radial structure diagrams, were analyzed through heuristic methods and subsequently submitted to student user testing. The evidence provided by the case study indicates a positive qualitative assessment from students regarding the purpose of the tool but are not yet conclusive regarding optimal morphological representation of the information.
\end{abstract}

Keywords: Visualización, Evaluación, Validación de modismos

\section{Introducción}

El presente estudio se sitúa en el contexto de una modalidad de evaluación -en desarrollo- para el taller de diseño (De la Sotta, 2012; 2013), denominada Output-Mapping (OM). Esta aplica un método que permite informar la evolución del alumno de manera más integral (Fig. 1). OM incorpora la evaluación con el enfoque de un modelo educativo en proceso de instalación institucional, el cual:

- Promueve la pedagogía centrada en el estudiante,

- Favorece métodos participativos activos,

- Adopta la formación orientada por competencias como elemento motriz del plan de estudios,

- Promueve estrategias metodológicas adecuadas y dispositivos de evaluación, para el desarrollo de competencias.

Los esfuerzos se centran en ajustar los prototipos anteriores creados por el profesor desarrollador del modelo de evaluación. Sin embargo, los avances del proyecto, llevan al equipo a enfrentar la necesidad de desarrollar nuevas propuestas (De la Sotta, Hamuy y Perelli, 2013). La generación de dichos prototipos es impulsada por tres preguntas: ¿Qué se presenta (nivel de datos), ¿Por qué se presenta (nivel de tarea) y ¿Cómo se presenta (nivel de presentación)? (Aigner, Miksch, Schumann y Tominski, 2011)

En una primera instancia la revisión de la literatura, deja en evidencia la existencia de varios otros recursos de visualización posibles, no considerados por el equipo docente del taller, además de la existencia de enfoques metodológicos para la evaluación y diseño de dichos modismos que visualizarán el proceso de enseñanza-aprendizaje del estudiante durante su tránsito por el taller. Una exploración mayor con el fin de determinar ¿cuál o cuáles recursos visuales hacen mayor sentido a los usuarios y son fidedignos al cuerpo de datos? (De la Sotta Lazzerini, 2014)

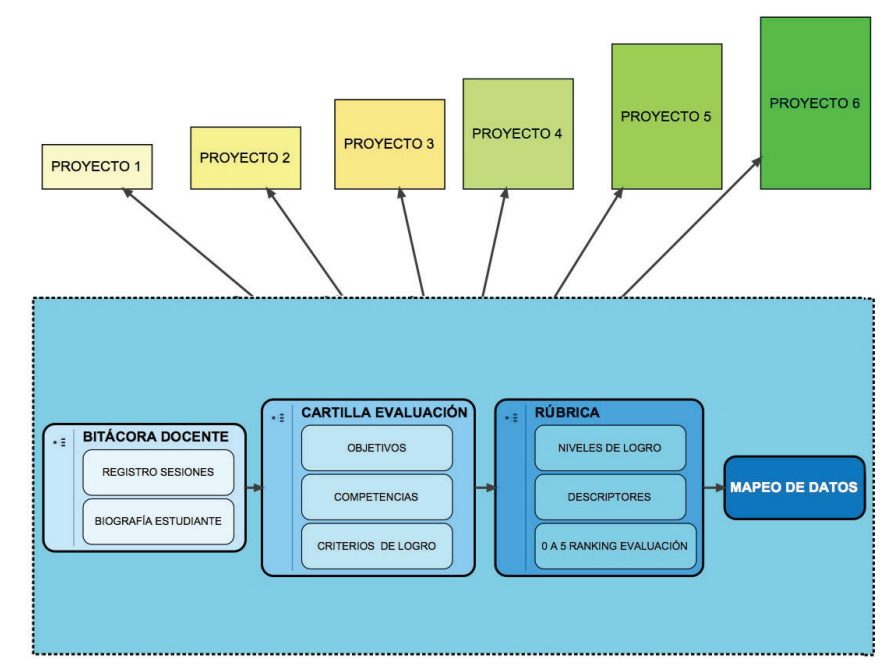

Figura 1. Modelo OM de evaluación. 
Se revisan estudios (Aigner et al., 2011; Burch y Weiskopf, 2014; Draper, Livnat y Riesenfeld, 2008; Tory y Moller, 2004; Ware, 2012) de las estructuras de datos o las morfologías visuales de representación a los que llamaremos modismos. (Munzner, 2014) Así, desde una perspectiva de High-level, se adopta la taxonomía modelo de diseño para el análisis, así como el enfoque de métodos mixtos. Ambos se ajustan a un enfoque de investigación basada en diseño. Desde una perspectiva Low-level, se abordan tipologías de modismos de visualización, centrando el foco en la comparación entre modismos cartesianos y radiales. Esta distinción se remonta a los modismos piloto utilizados, que evolucionan de formas cartesianas (líneas de tendencias, gráficos de barra), a formas radiales (gráficos de torta, gráficos polares y gráficos de radar). Draper et al. (2008) -por un lado- proporcionan un panorama muy completo y detallado de los diagramas radiales. Por otro lado, Burch y Weiskopf (Burch y Weiskopf, 2014) comparan las fortalezas y debilidades de estas dos tipologías generales de representación. Sin embargo, la validación de modismos de visualización fue abordado con poca profundidad en su momento.

El avance específico que aquí se presenta (Fig. 2), da cuenta de los resultados de someter a validación de usuarios, alternativas de modismos de visualización (Munzner, 2014), para representar las evaluaciones obtenidas por estudiantes, durante un semestre de la aplicación de OM. En trabajos anteriores (De la Sotta, Hamuy y Perelli, 2013; De la Sotta, 2012; 2013; Perelli, Hamuy y De la Sotta, 2014; Hamuy, Perelli y De la Sotta, 2015), se describe con mayor detalle los alcances pedagógicos de OM, las fases iniciales de revisión de la literatura y elaboración de los primeros prototipos de visualización.

\section{Procedimientos Metodológicos}

Los modismos de visualización de datos deben cumplir tres criterios encontrados en la literatura (Aigner et al, 2011; Munzner, 2014.): Expresividad, Efectividad y Adecuación. La Expresividad se refiere al requisito de mostrar exactamente la información contenida en los datos; nada más y nada menos debe ser visualizada para una correcta correspondencia de los datos. Efectividad considera principalmente el grado en que la visualización ocupa las capacidades cognitivas del sistema visual humano, además de la tarea en cuestión, los antecedentes de aplicación, y otra información relacionada con el contexto, para obtener representaciones visuales intuitivamente reconocibles e interpretables. Por último, la Adecuación implica una relación costo-valor con el fin de evaluar el beneficio del proceso de visualización con respecto a la consecución de una tarea determinada (Aigner et al., 2011: 3-4).
En etapas previas, la investigación es impulsada por lograr un ajuste técnico, un enfoque bottom-up o inductivo, a la visualización definida de modo intuitivo, sin embargo se transforma en una indagación impulsada por el problema de base, es decir de tipo top-down o deductivo. Para Munzner (2014), el diseño de visualización es una tarea difícil debido a la gran cantidad de posibilidades de diseño que pueden ser no efectivas, siendo un número muy reducido las opciones que realmente se ajustan al contexto de uso específico. Advierte que elegir posibilidades de visualización de manera aleatoria es una mala idea debido a que la probabilidad de encontrar una solución adecuada es muy baja.

Siguiendo este criterio, se opta por trabajar con una muestra intencionada de los 17 prototipos diseñados. Basados en las evaluaciones heurísticas hechas durante el proceso de desarrollo de los modismos, se seleccionan tres representativos de las categorías principales de la revisión teórica y la evaluación heurística: Gráfico Polar de Área (GPA), Gráfico de Barra (GB) y Gráfico Polar de Columna (GPC).

GPA es un modismo que durante la socialización con pares recibió retroalimentación positiva, valorado como "atractivo y novedoso". El modismo GB, es elegido debido a que culturalmente es conocido y no debía ser descartado a priori dado que su tipología cartesiana presenta una serie de atributos de expresividad y efectividad. Finalmente, el modismo GPC es elegido pues transfiere algunas de las fortalezas de los gráficos cartesianos a un eje polar. Los modismos fueron diseñados con la intención de ser presentados a los estudiantes vía WEB, basados en la idea inicial de la Profesora desarrolladora de OM de que éste fuese un sistema de consulta en línea de modo tal que el estudiante pudiese hacer la consulta de su progreso en el paso del taller desde cualquier lugar, en cualquier momento $y$, al mismo tiempo, poder compartir o descargar dicha información con mayor facilidad.

Para dicha tarea, hicimos pruebas preliminares con distintas librerías javascript ideales para el desarrollo de modismos de visualización para WEB. Entre estas se encuentran D3.js, Google Charts, Flow, entre otras. Finalmente, optamos por trabajar con Highcharts. Highcharts es una librería Javascript para la generación de gráficos. Fue lanzada en el año 2009

por la empresa Highsoft. Dentro de las razones por las cuales elegimos esta librería por sobre las librerías antes mencionadas, es el soporte para gráficos radiales, que nos permitiría rápidamente recrear el modelo inicial de visualización planteado por la profesora que desarrolló el método de evaluación, además, al ser gratuito y de código abierto, la posibilidad de bifurcaciones (fork), del código ofrece variados soportes y soluciones a través de plataformas como Github. 


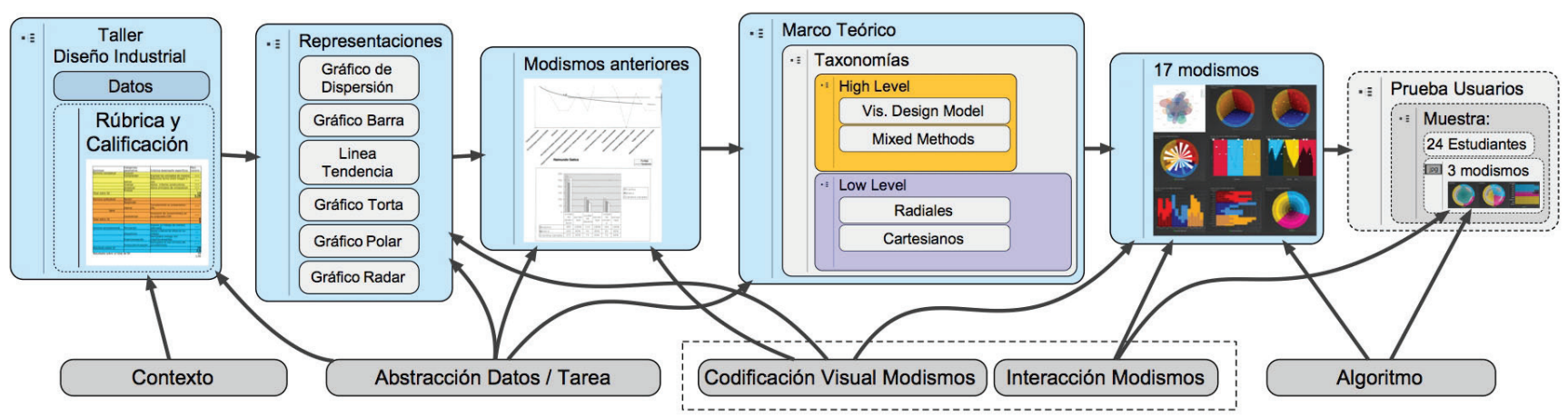

Figura 2. Fases de desarrollo del artefacto de visualización.

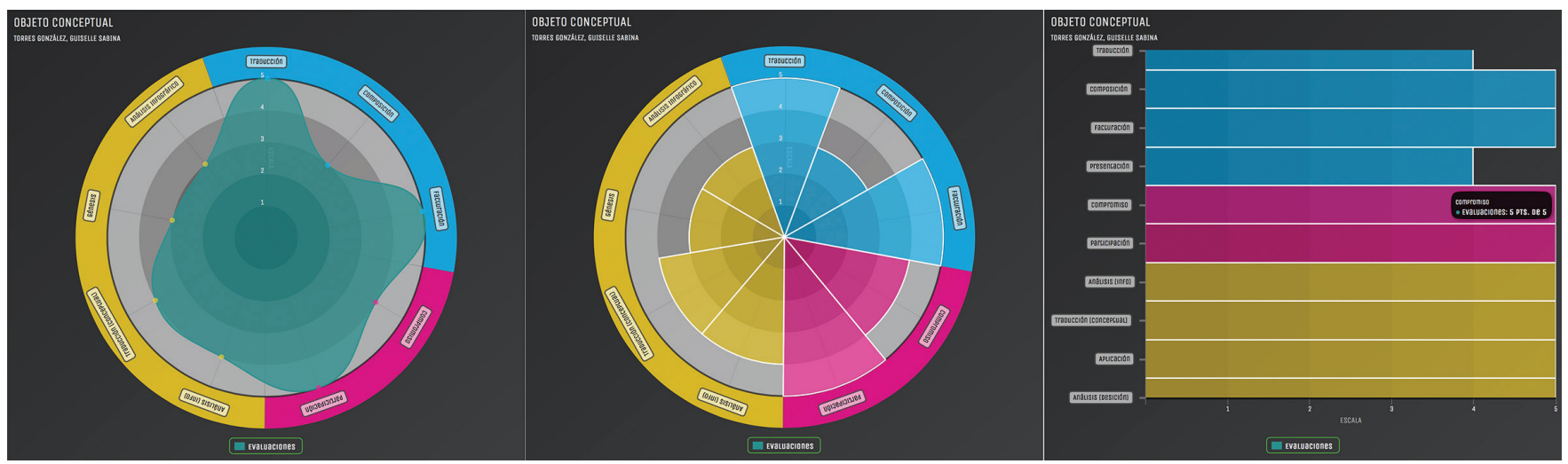

Figura 3. Modismos sometidos a pruebas de usuario: GPA, GPC y GB.

Quizá la principal razón por la que nos inclinamos por esta librería, es la integración entre HTML5, Javascript y la representación de elementos vía SVG (Scalable Vector Graphics), que la librería utiliza, de esta manera, la visualización no está sujeta a problemas de pixelación, situación que podría alterar la correcta lectura de líneas o textos además de ofrecer la posibilidad de animaciones mediante interpolaciones.

Quizá, la única limitante detectada para Highcharts es que de los modismos detectados durante el proyecto, solo es capaz de generar representaciones en grafos del tipo cartesianos y radiales.

Para la sesión de socialización con estudiantes, elaboramos fichas personales para cada uno de los integrantes del taller, compuesto de veintisiete estudiantes de segundo año de Diseño Industrial. Dichas fichas constaban de:

- Una rúbrica con los puntajes obtenidos durante los tres proyectos desarrollados durante el primer semestre, totalizando 36 sesiones en 18 semanas de clases.

- Dichos puntajes (datos) fueron ingresados en los tres modismos seleccionados de modo tal que los estudiantes vieran reflejados sus evaluaciones y puntajes de tres maneras distintas.

- Finalmente, a partir de sus interacciones con los tres modismos de visualización GPA, GB y GPC (Fig. 3), los estudiantes deberían contestar una encuesta compuesta de ocho preguntas abiertas en las que pudiesen expresar detalladamente sus impresiones, críticas y comentarios a partir del uso y lectura de sus evaluaciones en los tres modismos antes mencionados. Las preguntas realizadas fueron las siguientes:

P1: ¿Qué relación le otorga a los colores utilizados?

P2: ¿Es clara la información que intenta entregar la herramienta de visualización?

P3: Desde el punto de vista del formato de presentación de la información (tipo de gráfico), ¿cuál de ellos te pareció de fácil lectura y comprensión? ¿Por qué?

P4: ¿Cree usted que el método de evaluación es un aporte para la comprensión de su proceso personal durante el tránsito por el taller, y son el reflejo de su trabajo?

P5: ¿Si pudiera sugerir alguna modificación o sumarle algo a esta herramienta, cuál sería? ¿Por qué?

P6: ¿Diría usted que la herramienta de visualización le permite reflexionar sobre su desempeño durante el periodo evaluado? P7: ¿Qué prefieres como sistema de evaluación? ¿por qué?

- Evaluación cualitativa: rúbrica metafórica

- Evaluación cuantitativa: nota de 1 a 7

- Evaluación OM: integración de ambas

P8: Respecto de las etiquetas y leyendas de texto presentes en los gráficos ¿Consideras que son claros? Sí/No Justifica tu respuesta.

Una vez obtenidos los resultados, el equipo notó de que algunas de las respuestas de los estudiantes parecían ser inconexas o poco serias con respecto a lo que se les estaba 
preguntando. Si bien, para ese momento, el enfoque de métodos mixtos resultó ser de mucha utilidad, no reparamos en profundizar el aspecto de validación de los modismos. Surgen las interrogantes de ¿Cómo validar los modismos de visualización? y ¿Por qué es importante validar?

Munzner plantea un modelo de validación (Fig. 4) de modismos denominado Nested Model for Visualization Design and Validation framework (Modelo anidado de validación). Su estructura anidada, permite identificar debilidades en el diseño de visualización desde cuatro estadios:

1. Situación de Dominio (Domain Situation): donde se consideran detalles de un dominio de aplicación para la visualización, en otras palabras, el contexto que motiva la visualización.

2. Tarea y Abstracción de Datos: este es el nivel en el cual se deben identificar tanto las preguntas como la información, en un lenguaje que sea pertinente a quienes tienen la necesidad de visualizar. El mayor problema en este nivel es que al diseñar el modismo, nos arriesgamos a mostrar información incorrecta que no es parte de lo que se busca visualizar.

3. Codificación Visual e Interacción con el Modismo: en el tercer nivel se decide a dar forma específica a la visualización a partir de la abstracción de tareas y datos (nivel 2), las distintas aproximaciones de diseño de visualización o modismos (Munzner, 2014).

4. Algoritmo: es el elemento (proceso, librería, código, etc.), computacional que permite generar la representación de visualización. Munzner advierte que un error de algoritmo se da rara vez y que, de ocurrir, se considera un problema de índole técnico. Llevado a nuestro caso de estudio, problemas de algoritmo serían problemas de conectividad a la plataforma o errores de carga de Highcharts por mencionar algunos.

Con respecto a las preguntas ¿Qué se presenta? (nivel de datos), ¿Por qué se presenta? (nivel de tarea) y ¿Cómo se presenta (nivel de presentación)?, Munzner establece que el modelo anidado, da respuestas a dichas preguntas a través de los niveles Tarea y Abstracción de datos (¿Por qué? / ¿Qué?), mientras que Codificación Visual e Interacción con el Modismo se hace cargo de la pregunta (¿Cómo?). De esta forma,

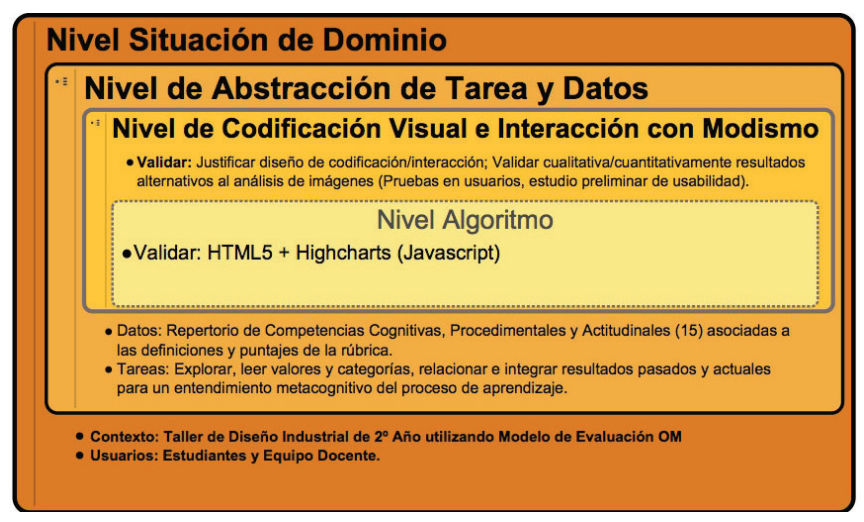

Figura 4. Modelo Anidado para Diseño y Validación de Visualización (Munzner, 2014). se optó por analizar nuevamente las preguntas realizadas a los estudiantes clasificándolas según el nivel de validación al que hacían alusión.

A partir de esta nueva organización, el cuestionario quedó dispuesto de la siguiente forma:

- Dominio: P7, P5

- Tarea y Abstracción de Datos: P1, P4

- Codificación Visual / Interacción con el modismo: P2, P3, P6, P8

- Algoritmo: Si bien este nivel no se abordó a través de las preguntas, la observación del comportamiento de los usuarios que interactúan en el laboratorio con los modismos, sirvió como un procedimiento de validación del algoritmo en general.

Al tener dispuestas las respuestas de los estudiantes de esta forma, la inconsistencia percibida en un momento, comenzó a disiparse, las respuestas por nivel de validación comenzaron a ser coherentes y complementarias entre sí.

\section{Resultados}

\section{Análisis del nivel Modismo de Codificación Visual / Interacción}

A continuación se han elegido algunos de los datos más relevantes obtenidos de la encuesta. Considerando que muchos de los ítems corresponden a preguntas abiertas, se procedió a hacer un análisis de contenido identificando las categorías del marco conceptual que pueden reconocerse presentes en las respuestas.

Tal como Mazza (2009) señala, el color es un atributo gráfico complejo, en la confluencia de los aspectos culturales, lingüísticos y fisiológicos. Las respuestas del usuario (Tabla 1) reflejan esta complejidad. La mitad de ellos $(11+1)$ relaciona los colores con aspectos culturales y lingüísticos, entre ellos estéticos. La otra mitad $(7+5)$ no reconoce ninguna relación.

\begin{tabular}{|l|c|}
\hline \multicolumn{2}{|c|}{ Cultural/lingüístico } \\
\hline Reconoce atributo cultural lingüístico & 11 \\
\hline No reconoce atributo cultural lingüístico & 7 \\
\hline Reconoce atributo estético & 1 \\
\hline No menciona atributo & 5 \\
\hline TOTAL Ordenado/categorial & 24 \\
\hline \multicolumn{2}{|c|}{} \\
\hline Reconoce atributo de orden cromático & 6 \\
\hline Reconoce atributo categorial: tono cromático & 16 \\
\hline No menciona atributo & 2 \\
\hline TOTAL & 24 \\
\hline
\end{tabular}

Tabla 1. P2: ¿Qué relación le otorgas a los colores utilizados?

Además, desde la expresividad y la efectividad analizadas por Munzner (2014), la gran mayoría $(6+16=22)$ reconoce atributos de orden o atributos categoriales en el uso del color. La mayoría (16) reconoce alta eficacia mediante Atributo 
Categorial / tono cromático. Otros (6) reconocen la efectividad de Atributo de Orden / gradaciones tonales.

Esta pregunta se refiere a las cualidades de presentación y las razones de las preferencias pueden estar relacionadas con su expresividad o su efectividad (Tabla 2). De los encuestados, 18 entradas se inclinaban a la GPA, 8 a la GPC y sólo una declaración alude a la apelación de la GB. Si se suman las entradas que se refieren a los dos modismos radiales, esto coincidiría con la afirmación de Burch y Weiskopf (2014) de qué gráficos radiales son más atractivos que los cartesianos para los usuarios.

\begin{tabular}{|l|c|}
\hline El más atractivo ... & 18 \\
\hline GPA & 8 \\
\hline GPC & 1 \\
\hline GB & \\
\hline Por qué... & 2 \\
\hline GPA Contribuye a la Expresividad & 16 \\
\hline GPA Contribuye a la Efectividad & 3 \\
\hline GPC Contribuye a la Expresividad & 5 \\
\hline GPC Contribuye a la Efectividad & 1 \\
\hline GB Contribuye a la Expresividad &
\end{tabular}

Tabla 2. P3: ¿Cuál de los formatos, barras ortogonales o radiales o superficies orgánicas te parece más atractivo y por qué?

En cuanto a las razones dadas por los usuarios de su preferencia, la efectividad de GPA pesa más; la expresividad tiene una referencia relativamente baja. GPC obtiene reconocimiento en cuanto a su expresividad y efectividad. Por último, el GB tiene una sola mención por su expresividad. En resumen, aunque la GPA se prefiere por su atractivo y -su efectividad-no destaca por su capacidad para hacer un mapeo preciso de los datos. En ese sentido, el GPC, que aunque tiene menos menciones, se distribuye de manera más uniforme entre la referencia a su expresividad y efectividad.

Aunque la P6 apunta a las cualidades de presentación, algunas de las respuestas sugieren la modificación de los datos o de la tarea, que se incorpore información o funciones. Con respecto a esto, los usuarios están de acuerdo en que los artefactos de visualización deben incorporar el factor tiempo de la evaluación, por ejemplo, ser capaz de comparar diferentes evaluaciones del año o de los años de carrera, y por lo tanto refuerzan la noción de proceso. Otros sugieren que la visualización también podría incorporar la retroalimentación del profesor sobre el trabajo del estudiante para complementar juicios evaluativos. Del mismo modo, los datos sugieren que las etiquetas adicionales podrían estar refiriéndose a la rúbrica de evaluación. También señala que este instrumento podría añadir campos para la evaluación por pares y la retroalimentación sobre el desempeño de estudiantes a los docente, por parte de los estudiantes.
En segundo lugar, otro grupo de sugerencias se refieren a aspectos de carácter formal. Son los comentarios (7), sugiriendo la necesidad de distintos ajustes a los elementos gráficos.

Hay tres comentarios que aluden a un error en la curvatura del gráfico de área polar. Durante la evaluación heurística de los prototipos, los investigadores ya notaron el error de expresividad en este modismo, lo que significa que en algunos casos los datos presentados en realidad no existen. Esto es porque el algoritmo genera contornos interpolados de curvas y contracurvas en base a datos que en realidad son discretos y no deben ser interpolados (Tory y Moller, 2004). Este error ya se había detectado en instancias anteriores de socialización el lenguaje con alumnos que reconocieron su funcionalidad y atractivos. Sin embargo por motivos técnicos, este juicio pareció como dudoso, por lo que fue sometido a pruebas de usuario.

\begin{tabular}{|l|c|}
\hline Etiquetas y Leyendas son claras & 6 \\
\hline Etiquetas y Leyendas son parcialmente claras & 9 \\
\hline Etiquetas y Leyendas no son claras & 9 \\
\hline TOTAL & 24 \\
\hline El Texto inclinado no es claro & 5 \\
\hline Se requieren ajustes gráficos & 9 \\
\hline $\begin{array}{l}\text { Etiquetas y Leyendas requieren complemento de } \\
\text { información }\end{array}$ & 4 \\
\hline Etiquetas y Leyendas son redundantes & 3 \\
\hline TOTAL & 21 \\
\hline Etiquetas y Leyendas son claras & 6 \\
\hline
\end{tabular}

Tabla 3. P8: Respecto de las etiquetas y leyendas de texto presentes en los gráficos ¿Consideras que son claros? Si/No Justifique su respuesta.

Por último, la P8también reporta del Nivel de Codificación visual del modismo, (Tabla 3) se centra en el uso de etiquetas y leyendas de texto en los modismos. Comentarios de los estudiantes indican que en estos elementos gráficos hay suficiente espacio para mejorar, ya que sólo 6 estudiantes están satisfechos con ellos y los 18 restantes se dividen entre aquellos que consideran el uso de texto parcialmente claro o creen que no es claro en absoluto. Las razones más mencionadas apuntan a las configuraciones gráficas, como en el análisis de P6. Uno en particular se destaca: el texto inclinado no es legible; confirmando lo que alguna literatura señala. Al añadir la información a los gráficos radiales “... tales como etiquetas de texto, surge la pregunta de si estas etiquetas deben radializadas, también (en lugar de seguir la orientación horizontal). Las etiquetas radiales de texto son difíciles de generar y son difíciles de leer, mientras que las etiquetas no radiales en un diagrama radial deterioran la estética" (Burch y Weiskopf, 2014: 448; traducción propia).

Hay tres entradas que indican que los subtítulos y leyendas serían redundantes. "Creo que son superfluos, gráfica 
bien desarrollada es capaz de transmitir todo lo necesario para el análisis" (Estudiante). Este punto de vista refleja un criterio de simplicidad que es a menudo presentado por diseñadores, pero sin embargo, si bien una consideración válida, no se puede tomar en el términos absolutos, ya que hay muchos comentarios de usuarios que piden más información también.

\section{Discusión}

La representación visual en la educación del diseño se ha trabajado extensamente como una herramienta para el desarrollo de la cognición en el diseño y también como una habilidad de la práctica profesional (Goel, 1995). Sin embargo, más específicamente, la visualización como un recurso de apoyo a la evaluación y la metacognición del proceso de aprendizaje parece ser un tema menos familiar. La visualización de datos e información es un campo de gran desarrollo hoy y atrae a diferentes disciplinas (psicología de la percepción, la informática y la ciencia en general, etc.), así como el diseño visual. Esperemos que este conocimiento pueda ser tratado con mayor profundidad en la enseñanza del diseño, tal como se propone.

Como Munzner podría preguntar: ¿El enfoque del diseño de visualización en este caso fue impulsado por el Problema o impulsado por la Técnica? El investigador que desarrolló OM en etapas anteriores, llegó previamente a un GPA intuitivamente, pero ese modismo de visualización no había sido validado de forma sistemática. Ese problema lanzó esta investigación, que fue inicialmente impulsada por la Técnica, a fin de mejorar la GPA. Las fases de investigación posteriores tuvieron como objetivo el desarrollo de un proceso de validación más sistemático que incluyó la GPA y la comparó con otros modismos, incluso el más convencional (GB).

Desde una perspectiva ex post facto, los cuatro niveles anidados de diseño de visualización -y validación- se levanta como un modelo conceptual sólido. Este modelo resultó muy útil para el análisis de los datos recogidos, las respuestas comenzaron a cobrar sentido y revelar, cómo ciertas características en las expresiones analizadas, muestran éxitos, pero también errores. También proporciona recomendaciones sensatas y directrices para mejoras en el futuro. Como ya se ha dicho, en la búsqueda inicial para modismos de visualización se basó principalmente en las necesidades de la tarea docente de los usuarios. Retrospectivamente, la exploración de modismos alternativos podría haberse beneficiado de la consulta previa sobre las expectativas de los estudiantes usuarios y haber levantado una descripción de su perfil (o perfiles).

Los resultados apoyan las siguientes consideraciones que deben examinarse en futuros trabajos.

Se requiere mayor diseño y una validación de mejores modismos. Los usuarios aprecian el idioma GPA para la efectividad, en relación con atributos atractivos como orgánico, suave, o por proporcionar una representación unificada de su proceso. Sin embargo, los investigadores desde su opinión de expertos (reforzados por algunos comentarios de los usuarios y la literatura) están convencidos de que este idioma tiene un defecto importante desde el punto de vista de su expresividad, porque a veces proporciona información que en realidad no se corresponde con los datos y es impreciso. Algunos de los beneficios de un GPA se puede encontrar en un GPC y, con reajuste adicional, podría ser puesto de relieve en el segundo. Pero GB, aunque reconocido como preciso, fiable (expresividad) y fácil de leer en términos de información (efectividad), es bajo en las preferencias y no ofrece una representación destacable de los estudiantes en su conjunto.

Muchas sugerencias de los usuarios también hacen hincapié en la necesidad de hacer visible información adicional que pueda ayudar a interpretar los gráficos y relacionarlos con su proceso en el curso del taller, tales como los criterios de rendimiento específicos de la rúbrica de evaluación o comentarios de los profesores.

Características complementarias también son sugeridas, como coevaluación de pares, retroalimentación o una perspectiva del factor tiempo con respecto a su proceso.

¿Qué puede la visualización contribuir a la evaluación en la enseñanza del diseño? El modelo de evaluación OM junto con el artefacto de visualización, pueden ayudar al estudiante a involucrarse más en su evaluación, haciendo los objetivos de aprendizaje más visibles y transparentes en el taller de diseño (desarrollo de las competencias de diseño o aprendizajes complejos) y del mismo modo, los criterios para la evaluación de la evidencia de este proceso de aprendizaje. Esto permitiría que los medios de evaluación fuesen parte de un proceso metacognitivo. Hasta el momento, no ha habido una evaluación de la posible influencia que puedan tener tanto el modelo OM como el artefacto de visualización en una mejora del aprendizaje de los estudiantes. Esta tarea queda pendiente para casos futuros, una vez que el artefacto esté en pleno funcionamiento y pueda ser aplicado regularmente durante un período significativo.

En buena parte de la literatura sobre visualización, se asocia este campo con la representación bases de datos masivas (también conocida como Big Data), a la implementación de un alto y complejo grado de interactividad y a consultas del tipo Data-Mining. Sin embargo, autores muy especializados, como C. Ware (2012), C. Chen (2013) y R. Mazza (2009) avalan una definición más amplia, que implica la representación visual sin considerar esencial estos tres aspectos. El proyecto considera que estos aspectos pueden ser una etapa más avanzada de la aplicación de la visualización con el artefacto. Los desarrollos preliminares presentados hasta el momento, están dirigidos al escalamiento en el futuro hacia bases de datos más robustas que puedan incluir grupos más grandes de estudiantes, que acumulen progresivamente el expediente de las evaluaciones del estudiante y sus evaluaciones a lo largo de su proceso formativo a lo largo de la carrera y, por lo tanto, permitir a los maestros y directivos de la escuela de diseño, y los estudiantes con respecto a sus compañeros, para interactuar con una visión integral en el tiempo y tal vez se complementan con otras herramientas de evaluación, tales como portafolios. 


\section{Agradecimientos}

Esta investigación contó con el apoyo del Concurso de Investigación FAU 2013, de la Facultad de Arquitectura y Urbanismo de la Universidad de Chile.

\section{Referencias}

Aigner, W., Miksch, S., Schumann, H., \& Tominski, C. (2011). Visualization of Time-Oriented Data. London: Springer London. doi:10.1007/978-0-85729-079-3

Burch, M., \& Weiskopf, D. (2014). On the Benefits and Drawbacks of Radial Diagrams. In W. Huang (Ed.), Handbook of Human Centric Visualization (pp. 429-451). New York, New York, USA: Springer New York. doi:10.1007/978-1-46147485-2

Chen, C. (2013). Mapping scientific frontiers: the quest for knowledge visualization. Retrieved from http://dx.doi. org/10.1007/978-1-4471-5128-9

De la Sotta Lazzerini, P. (2014). Output Mapping. "Modelo de evaluación del proceso de enseñanza aprendizaje en la asignatura de Taller." In O. Echevarría (Ed.), Congreso Latinoamericano de Enseñanza del Diseño. Actas de Diseño (Vol. 8, p. 34). Buenos Aires, Argentina: Universidad de Palermo. Retrieved from http://fido.palermo.edu/servicios _ dyc/publicacionesdc/archivos/478_libro.pdf

De la Sotta Lazzerini, P., Hamuy Pinto, E., \& Perelli Soto, B. (2013). Diseño de Aplicación en Base a Modelo de Evaluación del Proceso de Enseñanza Aprendizaje en El Taller Introducción Metodología Resultados / A Design Studio Course Assessment Model Applied. In M. Bernal \& P. Gómez (Eds.), SIGraDi 2013. Proceedings of the 17th Conference of the Iberoamerican Society of Digital Graphics: Knowledgebased Design (pp. 547-550). Valparaiso, Chile: USM Editorial. doi:10.5151/despro-sigradi2013-0106

Dela Sotta,P.(2012)Output Mapping. Documento presentación defensa para optar a Magíster en Didáctica Proyectual, Univ. del Bíobío, Concepción, nov. 2012. (Comunicación personal). De la Sotta, P. (2013) Output Mapping: Modelo de evaluación del proceso de enseñanza aprendizaje en la asignatura de Taller. IV Congreso de Enseñanza del Diseño Univ. de Palermo. 29-31 julio, B. Aires: Argentina

Draper, G. M., Livnat, Y., \& Riesenfeld, R. F. (2008). A survey of radial methods for information visualization. IEEE Transactions on Visualization and Computer Graphics, 15(5), 759-76. doi:10.1109/TVCG.2009.23

Goel, V. (1995) Sketches of thought MIT Press, Cambridge, MA Hamuy, E., Perelli, B., \& De la Sotta, P. (2015). Visualization as Assessment in Design Studio Courses. In R. Vandezande, E. Bohemia, \& I. Digranes (Eds.), Proceedings of the $3 \mathrm{rd}$ International Conference for Design Education Researchers (pp. 1481-1497). Chicago, USA: Aalto University. doi:10.13140/ RG.2.1.2642.5440

Mazza, R. (2009). Introduction to Information Visualization. Business (p. 139). Springer-Verlag London. doi:10.1007/978-184800-219-7

Munzner, T. (2014). Visualization Analysis \& Design (p. 428). CRC Press.

Perelli, B., Hamuy, E. y De la Sotta, P. (2014) Identificación de Recursos Digitales de Visualización para la Aplicación de un Modelo de Evaluación en el Taller. En XVIII Congreso Iberoamericano de Gráfica Digital SIGraDi, 12-14 noviembre, Universidad de La República, Montevideo, Uruguay. Blucher Design Proceedings, Diciembre de 2014, vol. 1, num. 8, p.586590. ISSN: 2318-6968. DOI: 10.5151/despro-sigradi2014-0121. Tory, M., \& Moller, T. (2004). Rethinking Visualization: A HighLevel Taxonomy. In Proceedings of the IEEE Symposium on Information Visualization (pp. 151-158). Washington, DC, USA: IEEE Computer Society. doi:10.1109/INFOVIS.2004.59

Ware, C. (2012). Information Visualization, Third Edition: Perception for Design (Interactive Technologies) (3rd ed.). Morgan Kaufmann. 